\title{
Caries management strategies by risk assessment-prevention and treatment
}

\author{
Kashish.J ${ }^{1}$, Dr. Iffat Nasim ${ }^{2}$ \\ ${ }^{I}$ (CRRI, Saveetha Dental College, Chennai, India. ) \\ ${ }_{2}^{2}(M D S$, SeniorLecturer,Saveetha Dental College, Chennai,India.)
}

\begin{abstract}
Traditional caries management consisted of the detection of carious lesions followed by immediate restoration. Placing a restoration doesn't guarantee a sound future for the tooth. On the contrary, it will be the start of the restoration cycle in which the restorationwill be replaced several times. So a different management strategy is recommended. Based on a proper diagnosis of caries taken into an account, thedynamics and the activity of the caries should be determined. Caries risk should be determined before treatment is considered. The treatment goals in caries management should be to prevent new lesions from forming and to detect lesions sufficiently early in the process so that they can be treated and arrested by non-operative means. Such management requires skill and are time consuming. Best practices dictate that once the clinician has identified the patient's caries risk, therapeutic and preventive plan should be implemented to prevent the pathological challenge posed by the caries risk factors. This article reviews the caries management strategies in prevention and treatment.
\end{abstract}

Keywords:Dental Caries, Flourides, Prevention, Risk Assessment, Risk Factors.

\section{Introduction}

Dental caries is the most common disease seen in dentistry. Regardless of the development in the field of dentistry it continues to be the world health concern. ${ }^{1}$ Dental caries is a transmissible infectious disease in which pathogenic risk factors prevail over protective factors producing demineralization of the tooth structure.If the disease is allowed to progress, surface cavitation and dental tissue destruction will result. This suggests that disease is a result of a shift in the homeostatic balance of the resident micro flora, due to change in the local environmental conditions that favors the growth of the cariogenic bacteria. Hence the management of the disease continues to be a challenge. Current science has determined that the key to dental caries management and disease treatment lies with modifying and correcting the complex dental biofilm and transforming oral factors to health. This can be attained through a best practice approach that reduces caries risk factors and increases caries protective factors. ${ }^{2-4}$

\subsection{Etiology Of Dental Caries}

\section{Discussion}

Factors involved in the caries process, which include tooth, dental plaque and diet was presented in 1960's in a model of overlapping circles. The model has been supplemented with the factors of time, fluoride, saliva and social and demographic factors.

\subsubsection{Dental Plaque}

High number of mutans streptococci and lactobacilli are probably the consequence of high sugar intake and the resulting periods of low $\mathrm{pH}$ levels in dental plaque. Streptococcus mutans are responsible for caries formation and initiation whereas lactobacillusfor its progression but dental plaque is cariogenic locally than these bacteria. Dental plaque is the poor predictor of high caries activity but absence of Streptococcus mutansare good indicators of low caries activity.

\subsubsection{Teeth}

Teeth consist of calcium phosphate mineral that demineralizes when essential $\mathrm{pH}$ lowers. As the $\mathrm{pH}$ of the oral cavity is restored dissolved calcium and phosphate reprecipitate on remaining mineral crystals. Remineralisation is slower than demineralization. If there is any disturbance during remineralisation then caries will progress and lesion will develop. Dentin is more vulnerable than enamel because of structural differences and impurities in the lattice.

\subsubsection{Diet}

Dietary carbohydrates are essential for the bacteria to produce acids that initiate demineralization. There was a certain correlation between sugar consumption and caries prevalence. 


\subsubsection{Time}

Time affects the carious process in many ways, when it is considered to be a chronic disease. Time indicates that the substrate must be available for a certain amount of time to cause demineralization. This gives the dentist and the patient enough time to plan for preventive treatment strategies.

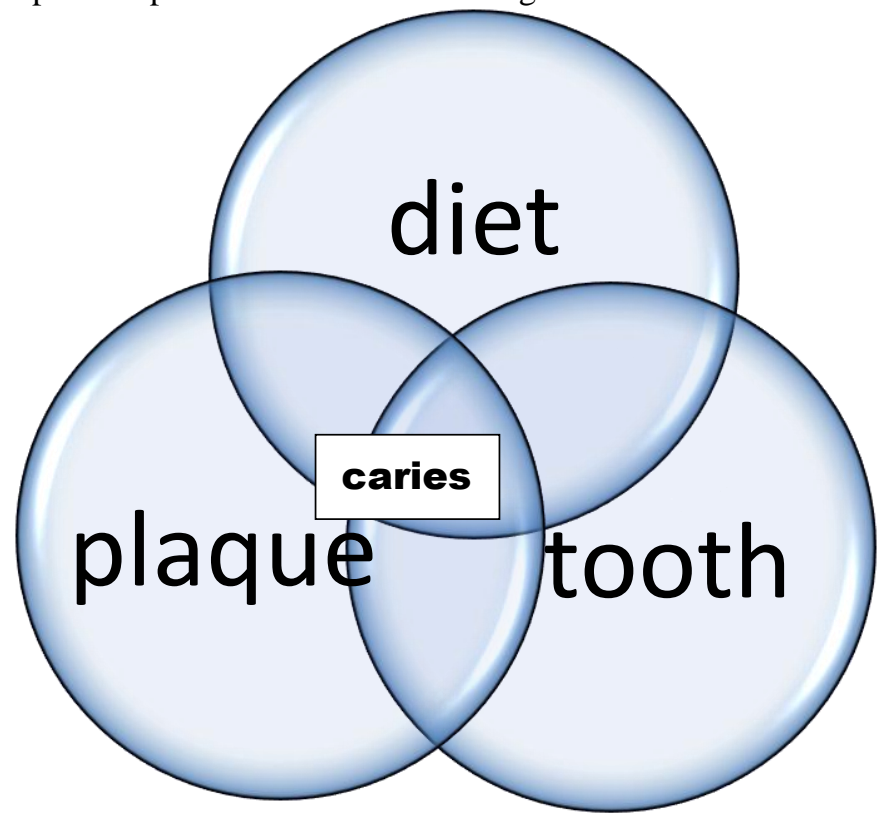

\subsection{Caries Risk Assessment}

Fig 1: Keye's Diagram

Caries risk assessment is a critical component of caries management. It should be included as a part of preliminary dental examination. Incorporation of risk assessment as a part of the examination provides better cost effectiveness and greater success rate when compared to the traditional management of patients irrespective of their risk.

The ADA developed two forms that determine low, moderate or high risk: one for patients 0-6 yr old and the other for patients older than six. Two caries risk assessment forms have been published in the journal of California dental association i.e., one for patients aged 0-5 yrs.' and other for patient's age through adulthood. The CDA forms are validated risk assessment forms which reveals statistically significant odds ratio relating to the future onset of cavitation. These forms differ in their risk factors, disease indicators and protective factors which strongly predict the future of the dental caries. Reassessment of the patients risk is considered as a best practice and it should be followed 3-12 months after the initial caries assessment with the interval of time depending on the risk level of the patients.

\subsection{Caries Balance Concept}

Caries balance concept represents multifactorial nature of the dental caries and emphasizes on the balance between the pathological and protective factors in the carious process. ${ }^{5,6}$ It is proposed by feather stone et.al in 2002.

\begin{tabular}{ll}
\hline Protective factors & Pathological factors \\
\hline Salivary flow and components & Reduced salivary function \\
$\begin{array}{l}\text { Proteins, } \\
\text { antibacterial components and agents }\end{array}$ & Bacteria: streptococcus mutans \\
$\begin{array}{l}\text { Fluoride, calcium phosphate } \\
\text { Dietary components: protective }\end{array}$ & Dietary components :frequent carbohydrate \\
\hline
\end{tabular}

Table 1

This a dynamic and delicate balance, tipping both sides many times a day. Progression or reversal of carious process is determined by the imbalance or balance between the disease indicators and risk factors on one side and completely protective factors on the opposite side. 


\subsubsection{Disease Indicators}

Disease indicators are described as physical signs of the presence of current dental caries or its history of activity. This indicator doesn't tell what has initially caused the disease or how to treat it but are strong predictors of the process. ${ }^{7}$ Caries balance concept uses acronym "WREC" to emphasize on these four disease indicators.

- White spots visible on smooth surface.

- Restoration placed in the last 3 yrs as a result of caries activity.

- Enamel approximal lesions visible on dental radiographs.

- Cavitation of carious lesions showing radiographic penetration into the dentin.

\subsubsection{Risk Factors}

Caries risk factors are biological reasons that promote future dental caries. Risk factors are based on the etiology of the disease. Risk factor can explain the imbalance that occurs when the disease exit. ${ }^{7}$ Caries imbalance model uses the acronym "BAD" to describe risk factors.

- Bad bacteria meanacidogenic, aciduric or cariogenic bacteria.

- Absence of saliva meaning hypo salivation or salivary hypo function.

- Destructive lifestyle habits such as ingestion of fermentable carbohydrates and poor oral hygiene.

\subsubsection{Bacteria}

Not all the oral bacteria are pathogenic. Cariogenic bacteria grow in large colonies and adhere to the tooth structure, the infermentable sugars from the carbohydrates turned into weak acids that cause demineralization. Carious lesion is a late stage symptom, so the evaluations of microbiological findings help the clinicians. Contemporary studies have shown distinct differences between the caries free and carious individuals. ${ }^{8} 9$

Streptococci mutans are part of normal flora which dominates due to its ability to produce intra and extra cellular polysaccharide that help with acid production and survival during low nutrition periods. Adherence to the tooth surface causes initiation of the caries. Lactobacillus is an acidogenic and aciduric bacteria that are found in low $\mathrm{pH}$ niches that are difficult to clean. ${ }^{10}$ It is more involved in the progression of the caries. ${ }^{11,12}$ Studies have found correlation between the mutans levels in the plaque biofilm and in saliva. ${ }^{13,14}$ Chair side tests are available to help clinicians to quantify Streptococcus mutans and lactobacillus levels in saliva. Culture based methods require tests that evaluates saliva bacteria. Culture findings higher than $10^{5} \mathrm{CFU}$ of MS/ LB indicates a high risk for caries disease. ${ }^{15,16}$

\subsubsection{Saliva}

The oral environment is regulated via the influence of salivary glands. Saliva is the only fluid in the mouth. It contains electrolytes such as sodium, potassium, calcium, magnesiumetc. ${ }^{17}$ This helps to modulate the bacterial attachment of the biofilm, buffering capacity of saliva and antibacterial properties.

Salivary gland hypo function or hypo salivation is the condition of reduced salivation that differs from the xerostomia i.e., complete oral dryness. Without adequate saliva, there is no proper clearance of sugary or acidic fluids and less urea to help clean plaque biofilm. ${ }^{18}$ The best way to determine the hypo salivation is to measure salivary flow. Determining saliva's quality, flow rate,viscosity,pH and buffering capacity helps the clinicians in preventive and therapeutic intervening as well as patient's education. A high salivary buffering capacity may result in an elevated $\mathrm{pH}$ of the enamel crystal resulting in favorable conditions for mineral uptake and remineralistions. ${ }^{19}$

\subsubsection{Diet}

Diet affects the $\mathrm{pH}$, quantity and quality of saliva, sugars and carbohydrates.After broken down by the enzymes, it helps oral bacteria to thrive and lowers the $\mathrm{pH}$ of the oral cavity. ${ }^{20}$ Development of carious lesion is reliable on the decrease $\mathrm{pH}$ which is a result of metabolism of dietary carbohydrates by oral bacteria. Fermentable carbohydrates gets digested by the salivary enzymes and fermented by oral bacteria. Simple sugars such as sucrose, fructose, and glucose are more cariogenic than complex carbohydrates. ${ }^{3}$

The frequency of meal and property of food influences the cariogenicity of patient's diet. The consistency and temperature of food can affect the oral clearance from the mouth. Oral sugar clearance is the reduction in the concentration of sugars in saliva and is known as the strong predictors of the prevalence of dental caries. Clinicians should realize that patients eating or dietary habits are dependent on many factorssuch as socioeconomic status, cultural ethnicity, cost and availability of food. ${ }^{21}$ At a minimum level, clinicians should asses for diet risk factors such as amount and frequency of the sugars and fermentable carbohydrates intakes and 
promote consumption of sugar substitute and health promoting snacks and meals. ${ }^{22,23}$ Recommendation of healthy snacks related to oral health will also help patient in reducing their risk level.

\subsubsection{Risk Age Groups}

\begin{tabular}{|c|c|}
\hline Age group & Features \\
\hline $1-2$ years & $\begin{array}{l}\text { Low educational background of parents } \\
\text { Mother with high MS levels. } \\
\text { Coincides with eruption of primary teeth. } \\
\text { Management: } \\
\text { Reduces sugar intake. } \\
\text { Reduce the microflora in mothers. }\end{array}$ \\
\hline $11-14$ years & $\begin{array}{l}\text { Coincides with eruption of second molars. } \\
\text { Most caries susceptible stage as enamel is undergoing } 2{ }^{\text {nd }} \text { maturation. } \\
\text { Management: } \\
\text { Integrated plaque control. } \\
\text { Remineralisation. }\end{array}$ \\
\hline Elderly adults & $\begin{array}{l}\text { Multiple restorations with plaque retentive margins, exposed root surfaces, } \\
\text { medications ,bad dietary habits, poor oral hygiene } \\
\text { Management: } \\
\text { Plaque control and remineralisation. }\end{array}$ \\
\hline
\end{tabular}

TABLE 2

\subsubsection{Preventive Factors}

Prevention or interception of all dental or oral diseases provides rehabilitation where disabilities have already occurred. Preventive strategies targeted at intercepting one or more of the necessary disease components of the Keye's diagram. In the 1950's and 60's caries prevalence was very high so then the prevention was most cost effective at the community level. The most plausible explanation for the decline of caries prevalence is the steady improvement in oral hygiene, which results in regular removal of dental plaque, combined with a regular daily administration of fluoride, provided via toothpaste. The cornerstone in any preventive strategy for the management of caries is oral self-care: twice daily careful cleaning of the tooth with a toothbrush and a effective fluoride toothpastes.

There are three levels of prevention. Primary, secondary and tertiary

\subsubsection{Primary Prevention}

The aim of the primary level of prevention is to prevent dental disease from happening. Primary prevention can be achieved on two levels: population and individual. The most accepted population approach in primary caries prevention is water fluoridation. Use of fluoridated toothpastes has also become common approach to caries prevention.

\subsubsection{Secondary Prevention}

Secondary prevention applies to cases where root or coronal surface decay has developed. Possibility of remineralisation of tooth surface with fluoride or the need for the replacement of the lost tooth structure should be assessed

\subsubsection{Tertiary Prevention}

Treatment of carious lesion involves far more than placing a restoration. Restorative treatments deal only with one consequence of the disease and not the causes. Restorative treatment will not be long lasting unless the disease causes are recognized and patient is equipped to be able to manage future decay challenges.

Based on caries balance concept :

Caries protective factors are biologic or therapeutic measures that can be used to prevent or arrest the pathological process of caries. ${ }^{7}$ The higher the risk factors, the greater the protective factors should be incorporated to reverse the process of caries. Protective factors enhance remineralisations and keep the balance 
between pathology and protection of the patient's oral health. The caries imbalance concept uses the acronym "SAFE" describes the following four protective factors

- Saliva and sealant.

- Antimicrobial or antibacterial agents

- Fluoride and other products that enhances remineralisations

- Effective life styles.

\subsection{Preventive Therapy Based On The Risk Status}

\begin{tabular}{|l|l|l|}
\hline Low risk & Moderate risk & High risk \\
\hline Fluoridated dentifrice & Fluoridated dentifrice & Fluoridated dentifrices \\
\hline & Systemic fluoride supplements & Systemic fluoride supplements \\
\hline & professional topical fluorides & Professional; topical fluorides \\
\hline & Sealants & Sealants \\
\hline & Antimicrobials & Home fluoride \\
\hline & & Dietary counselling \\
\hline
\end{tabular}

\subsubsection{Fluoride}

The use of fluoride is a cornerstone in prevention. A Cochrane review on fluoride confirmed the benefits of daily tooth brushing with fluoridated toothpaste to decrease or prevents dental caries in which atleast $1000 \mathrm{ppm}$ fluoride is present. ${ }^{24}$ Research emphasizes the positive use of fluoridated toothpastes, other topical fluoride modalities such as gels, varnishes, mouth rinses etc has been studied and confirmed their effectiveness. ${ }^{25}$ The American dental association council on scientific affairs developed evidence based guidelines for professional topical application of fluoride i.e., the application of fluoride gels and varnishes. The use of $5000 \mathrm{ppm}$ prescription of fluoride toothpastes and home use fluoride rinses has been recommended.

Fluoride varnish is a concentrated topical fluoride designed to stay in contact with the tooth surface for a long time enhancing fluoride uptake during early stages of demineralization. The efficacy of fluoride varnish is well studied and found to be more effective than the traditional fluoride gels. Its ease of use, relative safety makes it suitable for prevention. The ADA clinical guidelines suggests that application of fluoride varnish in two or four times in a year are effective in reducing carious lesions in children and adolescents at high risk for caries and the CAMBRA [clinical guidelines recommend a frequency of application of fluoride varnish as indicated by the patients caries risk. ${ }^{26-28}$

\subsubsection{Saliva}

Protection provided by saliva to the oral cavity is often overshadowed. An evaluation of the quantity and quality of saliva should be done at regular intervals for changes. Clinically the viscosity and the flow should be examined. Saliva is $99 \%$ water and should look like water, not thick and stringy or frothy and bubbly. ${ }^{29}$ According to the clinical guidelines, saliva testing should be done for all new patients and high levels of bacteria are suspected for patients who are at moderate risk for caries. High and extreme risk patients should be recommended for salivary testing at every recare examination. ${ }^{26}$

Healthy saliva is supersaturated with minerals calcium and phosphate, as $\mathrm{pH}$ of saliva drops the supersaturation of calcium and phosphate also drops, thus risk of demineralization. Remineralisation process deposits calcium and phosphate ions back into the tooth minerals forming new dentinal mineral which is stronger and more resistant to acid attack.

\subsubsection{Sealants}

Sealants are universally recognized as evidence based method to boost the tooth s resistance to carious lesions in pits and fissures of teeth. Until the sealant remains in pits and fissures, carious lesions will not occur, so it is important that clinician evaluate sealant retention on periodic visits. ${ }^{30}$ Unfilled and filled resin materials are available in the market. Fluoride releasing sealants are popular because of its fluoride releasing property from the sealant which helps in remineralisation and prevents carious lesions at the sealant margins. Glass ionomer cements suggested to be used as a sealant due to its fluoride release and hydrophilic nature but due to its poor retention rate with that of the resin bonded sealants its use is limited as transitional sealant on smooth surfaces. ${ }^{31,32}$

According to CAMBRA clinical guidelines, the placement of sealants is based on the risk of the patients. For low risk patients, resin based and glass ionomer sealants are optional. For moderate, high and extreme risk patients, pit and fissure sealants are recommended. Use of fluoride releasing sealants for deep pit and fissures has been emphasized in the new pediatric guidelines published in $2010{ }^{26,27}$ 


\subsubsection{Antimicrobials}

Antimicrobial agents surpass or destroy the growth or multiplication of microorganisms. CAMBRA clinical guidelines recommend the use of antimicrobials for patients over the age of six years classified at being high or extreme risk for caries and for caregivers of non-compliant moderate through extreme risk children under the age of six. ${ }^{26,27}$ Antimicrobials require repeated application at regular intervals, chlorhexidine gluconate rinse is FDA approval to treat gingivitis is effective in reducing the levels of mutans bacteria but is not effective against lactobacillus. Long term use of $0.12 \%$ chlorhexidine gluconate rinse in caries management has been questioned. ${ }^{33}$ The side effects of the chlorhexidine rinse are avoided by using chlorhexidine varnish. Chlorhexidine varnish approved for desensitization shown to be effective against cariogenic bacteria especially streptococcus mutans. It has shown most prevalent reduction of mutans. The application of varnish reduces the incidence of root carious lesions in geriatric populations. ${ }^{34,35}$ The application of chlorhexidine varnish every three to four months may be a more viable option especially for caregivers of children.Chlorhexidine gel is the next most effective followed by oral rinses for patients at moderate risk

\subsubsection{Xylitol}

Xylitol is generally accepted as naturally occurring sugar alcohol that reduces the amount of mutans and quantity of plaque biofilm when considered regularly. Studies have shown that consumption of xylitol reduces the transmission and colonization of mutans. ${ }^{36}$ Xylitol is dose dependent and minimum amount is needed to be effective about 5-6 gms /day divided into 3-4 doses for no shorter exposure than 5-10 min per exposure. ${ }^{37}$ CAMBRA clinical guidelines recommend the use of xylitolto control the cariogenic bacteria for patients over six years of age who are classified as moderate to extreme risk for caries. ${ }^{26,27}$ For children under six, xylitol wipes and products to replace sugary treats are recommended for children. Clinicians need to know the amount of xylitol present in the product because simply telling the patients to use xylitol gums three to four times a day may not be effective. The 2007 clinical guidelines for patients over six years of age recommend no more than $6-10 \mathrm{~g} / \mathrm{day}$.

\subsubsection{Effective Lifestyle Habits}

Use of fluoride has decreased the need for strict dietary control of sucrose. Caries doesn't occur in the absence of fermentable sugars so reducing the amount and frequency of sugar consumption remains important for patients at high risk. ${ }^{38}$ Consuming snacks or food that does not promote the progression of the carious lesion is ideal for those at high risk. Hard cheese has shown to coat teeth with a lipid layer protesting from acid attack. Studies show that arginine rich proteins in diet can increase the plaque PH rapidly.

Dental products that can assist in neutralizing acid and encourage non-acidic environment can be found in commercially available toothpastes and rinses. Chewing gums especially xylitol can raise plaque $\mathrm{pH}$ and reduce mutans at the same time. ${ }^{39}$ Calcium phosphate also raises plaque $\mathrm{pH}$ in addition to delivering calcium and phosphate to enhance remineralisation. Many calcium phosphate modalities has been introduced. Sugar free chewing gum containing casein phosphopeptide- amorphous calcium phosphate has shown increase in remineralisation. ${ }^{40}$

A CAMBRA clinical guideline > 6yrs of age suggest the use of the calcium phosphate for patients with excessive root exposure or sensitivity and is recommended for use several times daily for extreme risk patients. ${ }^{26,27}$ For pediatric patients the guidelines suggest that alternate brushing between toothpastes and calcium phosphate for moderate and extreme risk patients. For patients with high or extreme risk, a power tooth brush may be beneficial. Researches show that sonic toothbrushes enhance fluoride effects on the plaque biofilm causing increased retention and delivery of fluoride to the tooth surface. For extreme risk patients the sonic tooth brush has shown to increase salivary flow and decrease the incidence of root caries.

\subsubsection{CPP-ACP}

CPP-ACP is a remineralising agent introduced in food and dental products in 1998.ACP when added to a system that is demineralized, rapidlyhydrolyze into HA, that rebuilds enamel and fills the defects. Non crystalline ACP is rapidly soluble in the oral environment. Under normal intraoral $\mathrm{pH}$, CPP stabilizes and crystallizes ACP and prevents it from dissolving. CPP-ACP is additive in nature. $0.5 \mathrm{w} / \mathrm{v} \%$ sol of CPP-ACP is equivalent to 500ppm of fluoride in its action.

\subsubsection{Laser}

Use of lasers is the latest development in the field of dentistry. FDA approved use of Er: YAG laser on teeth in 1997. $\mathrm{Co}_{2}$ laser treatment inhibits caries progression. Kantorowitz et al stated that on irradiation of $\mathrm{Co}_{2}$ laser , carbonate is lost from the CAP tooth mineral, making it highly resistant to dissolution of acids. Laser 
would be set first to remove a minimum amount of carious tissue, then on the walls and base are treated to inhibit subsequent caries progression.

\subsubsection{Ozone}

Ozone is a powerful disinfectant. It is highly unstable. Carious enamel and dentin are permeable to ozone. It deactivates $99 \%$ of bacteria by release of radical ions. Lynch et al in 1987 stated that ozone breaks up the acidic products of cariogenic bacteria and promotes remineralisation without damaging adjacent tooth structure in 4-12 weeks. It is mainly used in the management of pit and fissures, smooth surface and root caries.

\subsubsection{Novamin Technology}

Novamin technology is a formulation of calcium, phosphate, sodium and silica. It is odorless, colorless and $100 \%$ biocompatible with the tooth structure. Novamin when exposed to saliva instantly releases billions of calcium and phosphate ions. Calcium and phosphate ions combine naturally occurring ions to form HA crystals. These ions also decreases inflammation and kill bacteria that cause gingivitis.

\section{Caries Control Restorations}

Caries control restorations are operative procedures where acute caries in multiple teeth are treated by removing infected tooth structure, medicate the pulp if necessary, and restore with a temporary material. In high risk individuals, along with the preventive procedures, restoration of the tooth structure is also indicated to repair the damage and reduce the caries risk of the individual. The objective of the caries control restoration is to limit further spread of caries and its possible sequelae. Direct pulp capping is most commonly advised. It is done only if tooth is vital before the procedure, exposure is less than $0.5 \mathrm{~mm}$, and the isolation of the tooth is perfect. If the lesion is deep, but not encroaching pulp, it is restored it with GIC or IRM of thickness 1-1.5 mm or greater. If a longer interval is anticipated then amalgam restoration is preferred. For the restoration of the tooth, precise anatomic form is not necessary but it should have proper proximal contacts and contours.

\section{Conclusion}

Modern day dentistry provides technical, esthetic and intellectual challenges to the dentist. Restorative dentistry is only one part of preventive dentistry. Noninvasive and preventive measures should be explored before initiating invasive procedures.One should keep in mind that caries is a dynamic process and its management requires the determination of caries activity and risk management. Assesment of the caries risk of the patient is an important factor in the caries management strategy. The ability of the clinician to motivate the patients and make them adhere to the clinician's instructions also plays a major role in caries management. Along with fluorides, new products are available to help clinicians with non-invasive caries management. So clinicians should carefully weigh the benefits and risks of recommending products or management strategies to their patients.

\section{References}

[1] Mouradian WE, Wehr E, CrallJJ.Disparities in childrens's oral health and acess to dental care. JAMA.2000;284(20):2625-2631.

[2] Young DA, Featherstone JD, Roth JR, Curing the silent epidemic: caries management in the $21^{\text {st }}$ centuryand beyond. J Calif Dent Assoc.2007;35(10):681-685.

[3] Marsh PD.Microbiology of dental plaque biofilms and their role in oral health and caries.DentClin N Am.2010;54:441-454

[4] Hara AT, Zero DT. The caries environment: saliva,pellicle, diet and hard tissue ultrastructure. Dent Clin N Am. 2010;54:455-467.

[5] Featherstone JD. The caries balance: the basis of caries management by risk assessment. Oral Health Prev Dent.2004;2(suppl 1):259-264.

[6] Featherstone JD, Domejean-Orliaguet S, Jenson L, Wolff M, Young DA. Caries risk assessment in practice for age 6 through adult. J calif dent assoc.2007;35(10):703-713.

[7] Young DA, Featherstone JBD. Implementing caries risk assessment and clinical interventions. Dent Clin N Am. 2010;54:495-505.

[8] Aas JA, Pastor BJ, Stokes LN, Olsen I, Dewhirst FE. Defining the normal bacterial flora of the oral cavity.J ClinMicrobiol. 2005;43:5721-5732.

[9] Corby PM, Lyons-Weiler J, Bretz WC, Hart TC, Aas JA, Boumenna T, Goss J,Corby AL, Junior AH, Weyant RJ, Paster BJ. Microbial risk indicators in early childhood caries.J ClinMicrobiol. 2005;43:5753-5759.

[10] Beighton D, S. Brailsford S. Lactobacilli and actinomyces: their role in the caries process; in: L. Stösser (Hrsg.) Kariesdynamik und Kariesrisiko; QuintessenzVerlags-GmbH, Berlin 1998.

[11] Van Houte J. Bacterial specificity in the etiology of dental caries. Int Dent J.1980;30(4):305-326.

[12] Kingman A, Little W, Gomez I, Heifetz SB, Driscoll WS, Sheats R, Supan P. Salivary levels of Streptococcus mutans and lactobacilli and dental caries experiences in a US adolescent population. Com Dent Oral Epidemiol. 1988;16:98-103.

[13] Hardie J, Thomson P, South R, Marsh P, Bowden G, McKee A, Fillery E, Slack G. A longitudinal epidemiological study on dental plaque and the development of dental caries - interim results after two years. J Dent Res. 1977;56:C90-98.

[14] Mundorff SA, Eisenberg AD, Leverett DH, Espeland MA, ProskinHM.Correlation between numbers of microflora in plaque and saliva. Caries Res.1990;24:312-317.

[15] Kneist S, Laurisch L, Heinrich-Weltzien R, Stösser L. A modified mitissalivarius medium for a caries diagnostic test. J Dent Res. 1998;77:970 (Abstr. 2712).

[16] Krasse B. Biological factors as indicators of future caries. Int Dent J. 1988;38:219-225. 
[17] Humphrey SP, Williamson RT. A review of saliva: normal composition, flow and function. J Prosth Dent. 2001;85(2):162-169.

[18] Dawes C. Salivary flow patterns and the health of hard and soft oral tissues. J Am Dent Assoc. 2008;139:18S-24S.

[19] Aiuchi H, Kitasako Y, Fukuda Y, Nakashima S, Burrow MF, Tagami J. Relationship between quantitative assessments of salivary buffering capacity and ion activity product for hydroxyapatite in relation to cariogenic potential. Aust Dent J. 2008:Jun;53(2):167171 .

[20] Touger-Decker R, van Loveren C. Sugars and dental caries. Am J ClinNutr. 2003Oct;78(4):881S-892S.

[21] Mobley C, Marshall TA, Milgrom P, Coldwell SE. The contribution of dietary factors to dental caries and disparities in caries.AcadPediatr. 2009;9(6):410-414

[22] Mobley C, Dounis G. Evaluating dietary intake in dental practices: doing it right. J Am Dent Assoc. 2010;141:1236-1241.

[23] Marshall TA. Chairside diet assessment of caries risk. J Am Dent Assoc.2009;140:670-674.

[24] Wong MC, Clarkson J, Glenny AM, Lo EC, Marinho VC, Tsang BW, Walsh T, Worthington HV. Cochrane Reviews on the Benefits/Risks of Fluoride Toothpastes.J Dent Res. 2011 Jan 19. [E-pub ahead of print]

[25] Marinho VC, Higgins JP, Sheiham A. One topical fluoride (toothpastes, or mouthrinses, or gels, or varnishes) versus another for preventing dental caries in children and adolescents. Cochrane Database Syst Rev. 2004;(1):CD002780.

[26] Jenson L, Budenz AW, Featherstone JDB, Ramos-Gomez FJ, Spolsky VW, Young DA. Clinical protocols for caries management by risk assessment. J Calif Dent Assoc. 2007;35(10):714-723.

[27] Ramos-Gomez F, Crystal YO, Ng MW, Crall JJ, Featherstone JDB. Pediatric dental care: prevention and management protocols based on caries risk assessment. J Calif Dent Assoc. 2010;38(10):746-761.

[28] American Dental Association Council on Scientific Affairs. Professionally applied topical fluoride: evidence-based clinical recommendations. J Am Dent Assoc.2006:137(8):1151-1159.

[29] Humphrey SP, Williamson RT. A review of saliva: normal composition, flow and function. J Prosth Dent. 2001;85(2):162-169.

[30] Ignelzi Jr. MA. Pit and fissure sealants - an ongoing commitment. J Calif Dent Assoc. 2010; 38(10);725-728.

[31] Sasa I, Donly KJ. Sealants: review of the materials and utilization. J Calif Dent Assoc.2010; 38(10);730-734.

[32] Beauchamp J, Crall JJ, Donly K, Feigal R, Gooch B, Ismail A, Kohn W, SiegalM,Simonsen R. Evidence-based clinical recommendations for the use of pit and fissure sealants. J Am Dent Assoc. 2008;138(3):257-268.

[33] Autio-Gold J. The role of chlorhexidine in caries prevention.Oper Dent.2010;33(6):710-716.

[34] Baca P, Clavero J, Baca AP, González-Rodríguez MP, Bravo M, ValderramaMJ.Effect of chlorhexidine-thymol varnish on root caries in a geriatric population: a randomized double-blind clinical trial. J Dent. 2009 Sep;37(9):679-685.

[35] Tan HP, Lo EC, Dyson JE, Luo Y, Corbet EF. A randomized trial on root caries prevention in elders. J Dent Res. 2010 Oct;89(10):1086-1090

[36] Söderling E, Isokangas P, Pienihäkkinen K, Tenovuo J. Influence of maternalxylitol consumption on acquisition of mutans streptococci by infants. J Dent Res.200;79:882-887.

[37] Twetman S. Treatment protocols: nonfluoride management of the caries disease process and available diagnostics. Dent Clin N Am. 2010;54:527-540.

[38] Burt BA, Pai S. Sugar consumption and caries risk: a systematic review. JDent Educ.2001;65(10):1017-1023.

[39] Duane B. Xylitol gum, plaque pH and mutans streptococci. Evid Based Dent.2010;11(4):109-110.

[40] Caruana PC, Mulaify SA, Moazzez R, Bartlett D. The effect of casein and calcium containing paste on plaque pH following a subsequent carbohydrate challenge. J Dent. 2009 Jul;37(7):522-526. 